\title{
The phosphorylation status of membrane-bound nucleoside diphosphate kinase in epithelia and the role of AMP
}

\author{
Kate J. Treharne • Oliver Giles Best • \\ Anil Mehta
}

Received: 7 January 2009/Accepted: 2 April 2009/Published online: 28 April 2009

(C) Springer Science+Business Media, LLC. 2009

\begin{abstract}
Nucleoside diphosphate kinase (NDPK) has many roles and is present in different locations in the cell. Membrane-bound NDPK is present in epithelial fractions enriched for the apical membrane. Here, we show in human, mouse and sheep airway membranes, that the phosphorylation state of membrane-bound NDPK on histidine and serine residues differs dependent on many regulatory factors. GTP (but not ATP) promotes serine phosphorylation (pSer) of NDPK. Further we find that rising [AMP] promotes pSer (only with GTP) but inhibits histidine phosphorylation (pHis) of NDPK from both donors. We find that NDPK co-immunoprecipitates reciprocally with AMP-activated kinase and that these two proteins can co-localise in human airways. AMP concentrations rise rapidly when ATP is depleted or during hypoxia. We find that, in human airway cells exposed to hypoxia (3\% oxygen), membrane-bound NDPK is inhibited. Although histidine phosphorylation should in principle be independent of the nucleotide triphosphates used, we speculate that this membrane pool of NDPK may be able to switch function dependent on nucleotide species.
\end{abstract}

Keywords Epithelia · NDPK · AMPK ·

AMP-activated kinase $\cdot$ GTP · CK2

\section{Introduction}

A number of studies have suggested that phosphorylation of nucleoside diphosphate kinase (NDPK or NM23) may

K. J. Treharne · O. G. Best · A. Mehta $(\varangle)$

Division of Medical Sciences, Ninewells Hospital and

Medical School, University of Dundee, Dundee DD1 9SY, UK

e-mail: a.mehta@dundee.ac.uk be important for function. For example, it was found that the wild-type NM23-H1 isoform suppressed the motility of cancer cells when over-expressed, but the NM23-H1 S120G or S120A mutants were unable to do so $[1,2]$. It was later reported that $\mathrm{S} 120$ is a target for phosphorylation by CK2, which inhibits the auto-phosphorylation of H118 [3]. This histidine phosphorylation was also found to be defective in the pancreatic islets of a diabetic (GotoKakizaki) rat model, which exhibits defective regulation of insulin secretion [4]. Islet cells are unusual in that insulin secretion in response to glucose does not require a glucose receptor at the cell membrane. Instead, the metabolism of glucose regulates insulin secretion. Protein phosphorylation by multiple kinases regulates this process and AMPactivated kinase (AMPK) plays a pivotal role [5]. Here we explore the potential interaction between NDPK and AMPK in an epithelial context.

An important difference between a non-polarised cell such as a fat or muscle cell and an epithelial cell lies in the separation of distinct domains of the plasma membrane. This difference occurs because epithelial cells face the external environment and have evolved apical membranes with different lipid and protein compositions compared to their inward facing basolateral membranes that are in contact with the interior spaces of the organism. These differences reflect the different functions required of the two membranes. To study epithelial membrane function, the purified apical membrane of the ovine tracheal epithelium has proved to be a useful resource and this led to our discovery of membrane-bound pools of nucleoside diphosphate kinase (NDPK, isoforms NM23-H1 and NM23-H2). It was found [6, 7] that this NDPK exists in at least two pools with different binding characteristics to the membrane. We discovered these NDPK pools whilst studying the relationship between the 
calcium binding protein annexin I, cAMP and histidine phosphorylation of annexin $I$ in the apical membrane of the tracheal epithelium. We found that cAMP inhibits the histidine phosphorylation of annexin I, and AMP was as effective an inhibitor as cAMP [8]. In that study we used EDTA to extract divalent cation-dependent proteins from the membrane. One NDPK pool (predominantly NDPK A, NM32-H1) could be extracted with EDTA whereas another remained tightly membrane-attached [6-9]. Since NDPK is reported to interact with cAMP [10, 11], we employed cAMP affinity chromatography and found that cAMP interacted differentially with these pools such that the EDTA-extracted pool, but not the membrane-retained (CHAPS-solubilised) pool of NDPK was able to bind. That these EDTA-extractable and membrane-retained NDPK fractions were indeed different was further exemplified when each pool was overlaid onto blots bearing the same membranes separated by SDS-PAGE (far-western technique). NDPK from the CHAPS-solubilised tracheal membranes was found to bind a $63 \mathrm{kDa}$ protein in addition to itself, but the EDTA-extracted fraction displayed no binding at this molecular weight. Attempts were then made to determine the identity and regulatory properties of this $63 \mathrm{kDa}$ protein, which led to our proposal that it was the $\alpha$ subunit of AMPK. However, these conclusions have recently been confounded by retraction of two papers from 2006 and 2007 (Crawford et al. Mol Cell Biol and FASEB J, respectively), which proposed a mechanism of kinase-kinase interaction between protein kinase CK2 (formerly casein kinase 2) [12], NDPK and the $63 \mathrm{kDa} \alpha$-subunit of AMPK [13-15]. Therefore, we have re-evaluated our previously unpublished data (from 2000-2002) and present this earlier evidence for an interaction between NDPK and AMPK here. These data represent observations made by KJT during her Wellcome Trust supported fellowship.

This article focusses on membrane-associated NDPK and considers the factors controlling the histidine autophosphorylation of this protein histidine kinase in the apical membranes of epithelia. We explore the phosphorylation of this membrane-bound fraction of NDPK and discuss its potential partners, factors influencing the level of NDPK phosphorylation and the relationship of the latter to the labelling of other membrane proteins. The regulatory mechanisms we have observed are conserved across three species (mouse, sheep, human). The data point to a link to [AMP], reinforce the likely interaction with AMPK and show that this NDPK, when in a membrane environment, can discriminate between ATP and GTP. Since this mechanism cannot be explained by in vitro enzymology (NDPK as a pure enzyme has no selectivity for the base moiety within the nucleoside); this suggests additional regulation of NDPK.

\section{Methods}

Briefly, we studied either (a) airway biopsies fractionated to enrich for apical membranes using discontinuous sucrose density gradient centrifugation (Treharne et al. 1994), (b) a cytosol free pellet (P4) of $\sim 6$ fold apically enriched sheep tracheal membranes which we previously used to purify NDPK and annexins [8], (c) plasma membranes purified from monolayers of a cultured human airway cell line (HBE [16, 17] or (d) various murine preparations of gut, lung and nasal airway epithelia. For the latter, mice were reared in ethically-approved facilities compliant with local legislation and euthanased by approved regulatory schedules. Bowel was flushed with saline, and the lumenal surface was scraped with a glass slide to harvest the epithelial cells. In each case, purified membranes were solubilised in MOPS-Triton buffers as described in [18], proteins separated by SDS-PAGE using lower gel buffer at either $\mathrm{pH} 8.9$ or 6.5 and phosphoproteins were visualised without acid treatment of gels by electronic autoradiography (Perkin Elmer, Instant Imager). For some studies on phosphohistidine (pHis) and phosphoserine (pSer) membranes were treated with acid $(0.1 \mathrm{M} \mathrm{HCl})$ or alkali $(0.1 \mathrm{M} \mathrm{NaOH})$ prior to running the gels and autoradiography.

A human bronchial epithelial cell line (16HBE14o-, HBE) was cultured on transwell membranes at air-liquid interface for three weeks at different $\mathrm{pO}_{2}$ levels before fixation for immunofluorescent analysis. Membranes with cells attached were stained using antibodies specific for NDPK and AMPK $\alpha 1$.

NDPK classically transfers $\gamma$-phosphates between nucleoside triphosphates and diphosphates. However, under the above phosphorylation conditions (no exogenous $\mathrm{Mg}^{2+}$ added, but in the absence of EDTA) at low temperature, the activity of membrane-bound NDPK was below the limit of detection (data not shown). To determine that NDPK could be active in these membranes, the rate of transfer of ${ }^{32} \mathrm{P}$ from $\gamma\left[{ }^{32} \mathrm{P}\right] \mathrm{ATP}$ (or GTP) to a GDP (or ADP) acceptor was measured at $37^{\circ} \mathrm{C}$ over the linear range (Fig. 1c). This activity is equivalent to GTP generation at $\sim 55 \mathrm{pmol} / \mu \mathrm{g}$ protein/min. It must be emphasised that this refers to total membrane protein. We confirmed that human airway cell membranes derived from nasal brushings could also transfer phosphate in this manner and similar transfer was observed for mouse and sheep airway epithelial membranes (not shown). Antibodies to NDPK were 


\section{A Ovine NDPK}

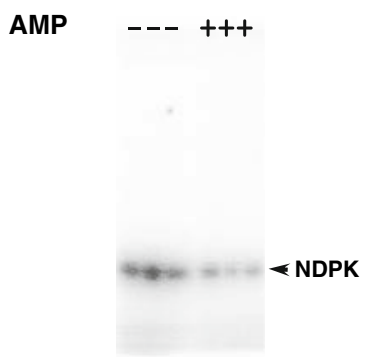

Human apical

membranes

AMP

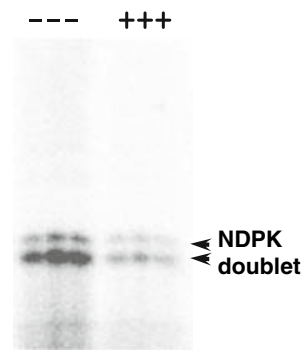

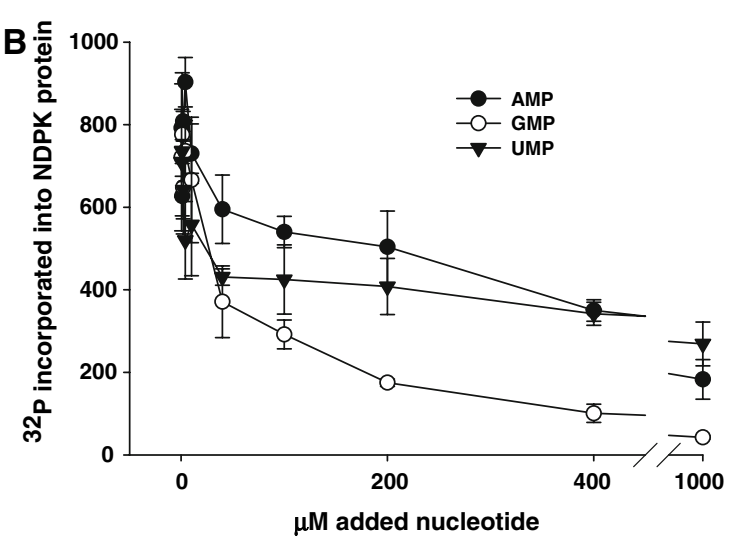

Fig. 1 Effect of nucleotides on NDPK phosphorylation. a Phosphorylation of NDPK at $4{ }^{\circ} \mathrm{C}$ is inhibited by $1 \mathrm{mM}$ AMP in both ovine (left panel) and human (right panel) membranes run in triplicate; NDPK typically shows up as a phosphorylated doublet in human membranes.

purchased from Santa Cruz. AMPK antibodies were a kind gift of D.G. Hardie, Dundee. All reagents were of analytical grade.

\section{Results}

NDPK is an early phosphoprotein (within one minute) labelled at $4{ }^{\circ} \mathrm{C}$ in airway membranes from human, mouse and sheep airway $[9,18]$. When a $6-15$ fold apically enriched epithelial membrane preparation of airway epithelium was incubated with $\gamma\left[{ }^{32} \mathrm{P}\right] \mathrm{ATP}$ (or GTP) at $4{ }^{\circ} \mathrm{C}$ for $\sim 1-5 \mathrm{~min}$, we observed only one pair of $\sim 18 \mathrm{kDa}$ phosphoproteins, which we showed to be isoforms $\mathrm{A}$ and $\mathrm{B}$ (NM23-H1/H2) of nucleoside diphosphate kinase (NDPK). Furthermore, the acid lability of this labelling suggested that the $\gamma$-phosphate had been transferred to phosphohistidine, to H118 in the active site [9]. Figure 1a shows that this phosphorylation is inhibited when the reaction is performed in the presence of $1 \mathrm{mM}$ AMP both in ovine (Fig. 1a, left panel) and human epithelial membranes (Fig. 1a, right panel). This inhibition was investigated further using human primary apical membranes and a dose response to AMP. These data show that the $\mathrm{IC}_{50}$ is around

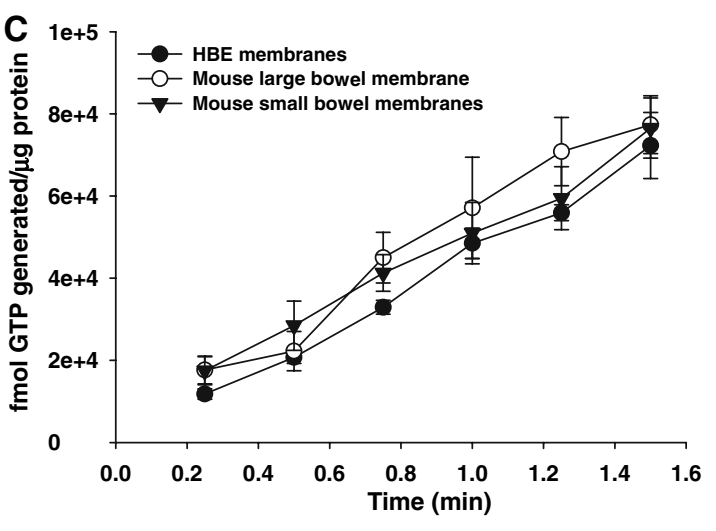

b Dose response of human NDPK phosphorylation to AMP, GMP and UMP. c Membrane activity (transfer of $\gamma$-phosphate from ATP to GDP) of NDPK from human (HBE cell line) and mouse gut epithelia

$200 \mu \mathrm{M}$ for AMP, similar for UMP, but is about $30 \mu \mathrm{M}$ for GMP. However, we observe that the inhibition occurs with a common rapid phase between 0 and $50 \mu \mathrm{M}$ for all nucleotides tested, followed by a much slower phase up to $1 \mathrm{mM}$. The potent effects of GMP were not explored further. To test the function of this membrane-associated NDPK, a human airway cell line (HBE), as well as epithelial membranes from murine gut were tested for NDPK activity. We observed that GTP was generated with similar kinetics from each membrane preparation and the rate was linear for $1.5 \mathrm{~min}$ (Fig. 1c).

In addition to the autophosphorylation of NDPK at H118, this protein can also be phosphorylated on a serine residue adjacent to the H118 [3], however, this study was conducted before the advent of mass spectrometry. To date, no absolute identification of this serine being phosphorylated has been performed. These serine and histidine phospho-amino acids may be distinguished by their distinct $\mathrm{pH}$ lability (only phosphohistidine is acid-labile in SDSPAGE gels buffered to $\mathrm{pH}$ 6.5). The upper panel of Fig. 2 shows that, when ATP is the phospho-donor, NDPK has no detectable acid-stable phosphorylation (i.e. no detected pSer at $\mathrm{pH}$ 6.5, implying that only pHis must have been present). In contrast, GTP as phosphate donor results in a 


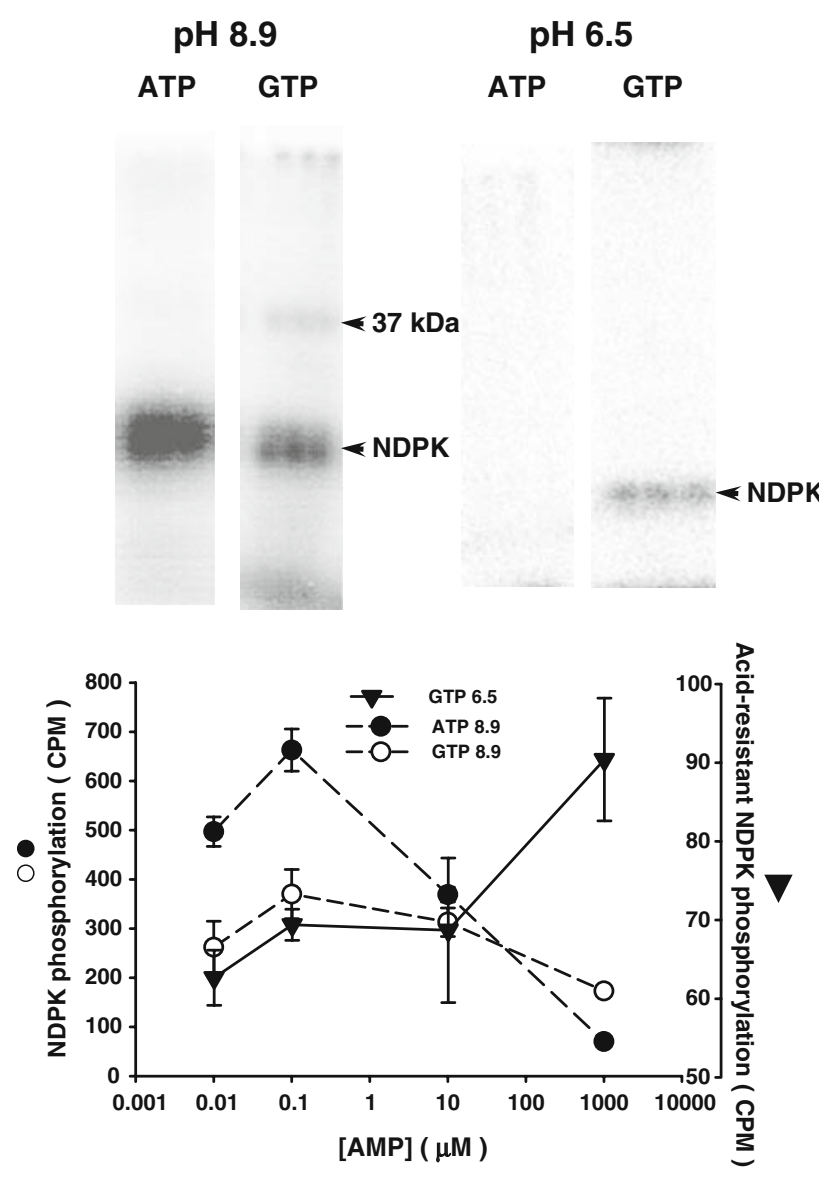

Fig. 2 SDS-PAGE of phosphorylated ovine NDPK run at two different resolving gel $\mathrm{pHs}$ in triplicate. No phosphorylated NDPK could be detected when ATP was the phosphate donor and the gel was run at $\mathrm{pH} 6.5$ (top right panel). Quantification of phosphorylation is shown in the lower panel. The $\mathrm{x}$-axis shows the effect of increasing [AMP] on the labelling of NDPK. Total phosphorylation is shown on the left axis (circles, dotted lines). Acid-stable phosphorylation is shown on the right axis, which is at a greatly expanded scale compared to the left (closed triangles, solid line), with GTP as the phosphate donor. There was no detectable acid-stable phosphorylation when ATP was the phosphate donor irrespective of [AMP] (not shown)

small amount of acid-stable labelling of NDPK. Quantification (Fig. 2, lower panel, note that the left and right $y$ axes are on different scales) shows that this acid-stable labelling is a quarter of the phosphorylation observed at $\mathrm{pH}$ 8.9. Furthermore, with increasing AMP concentration, this acid-stable labelling increases by fifty percent, despite the total phosphorylation (at the same time) declining to ten and sixty percent for ATP and GTP respectively.

AMP has many cellular effects, but it is increasingly recognised that AMP-activated protein kinase (AMPK) mediates many of these $[15,19]$ and its catalytic subunit has a molecular weight of $63 \mathrm{kDa}$. We therefore investigated whether AMPK could affect the NDPK studied in these experiments. Figure 3 shows that the addition of
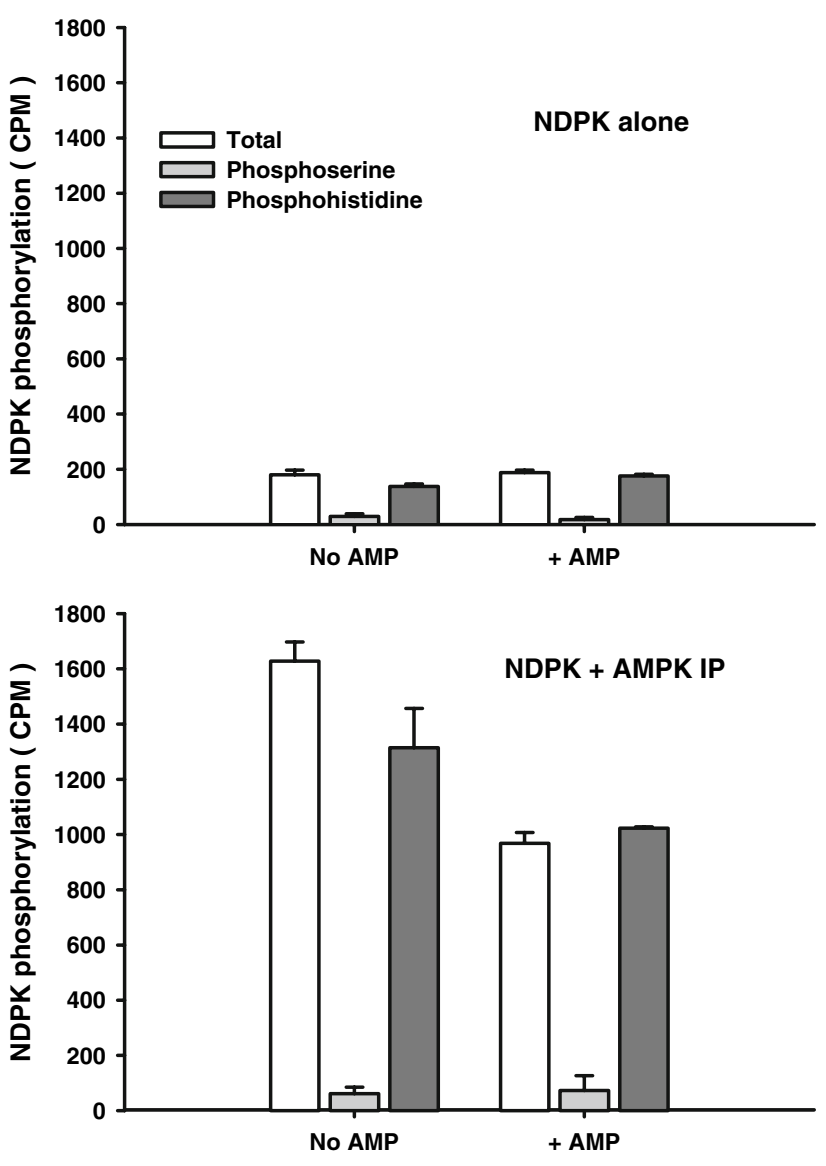

Fig. 3 Addition of AMPK to an NDPK-containing fraction enhances NDPK phosphorylation. Upper and lower panels show NDPK phosphorylation in the absence and presence of AMPK respectively. Addition of AMPK enhanced NDPK phosphorylation (compare open bars, upper and lower panels). $25 \mu \mathrm{M}$ AMP has no effect on baseline NDPK phosphorylation but attenuates the rise when AMPK is present (compare left and right datasets, lower panel). Acid/base ( $\mathrm{pH} 2 / 11)$ treatment of the gel samples was used to discriminate between baselabile phosphoserine and acid-labile phosphohistidine

immunoprecipitated AMPK increased the total $(\sim$ ninefold), histidine ( $\sim$ nine-fold) and serine (six-fold) phosphorylation of NDPK in the absence of AMP. In the presence of AMP, this increase was attenuated (5-fold). Serine phosphorylation was negligible relative to pHis. We interpret these data to suggest that AMPK, independent of AMP, can augment NDPK (auto)phosphorylation and may enhance its serine phosphorylation (dependent on the nucleotide donor). In contrast, the addition of AMP makes AMPK less able to augment the autophosphorylation of NDPK. Although these data are apparently in conflict with each other, they nevertheless imply that the two proteins may interact in both AMP-dependent and independent modes and this was tested in different ways. We showed that the two protein kinases co-immunoprecipitate reciprocally from ovine airway membranes and HBE cell membranes (Fig. 4, panels a + b). In addition, Fig. 4c (top 
Fig. 4 Association of NDPK with AMPK. a

immunoprecipitation from ovine tracheal extract using an anti-NDPK antibody, probed for AMPK $\alpha 1$ (upper panel) and immunoprecipitation using anti$\beta$ AMPK probed for NDPK (lower panel). b immmunoprecipitation from solubilised HBE membranes using anti- $\beta$ AMPK probed for NDPK. c Top panel shows quantification of NDPK phosphorylation in HBE membranes with or without prior metformin treatment of intact cells ( $2 \mathrm{mM}, 15 \mathrm{~min})$. Bottom panel; Nasal brushings from healthy volunteers were incubated in medium $199 \pm 600 \mu \mathrm{M}$ AICAR for $90 \mathrm{~min}$ at room temperature before phosphorylation of cell lysates. Quantification of NDPK phosphorylation is shown
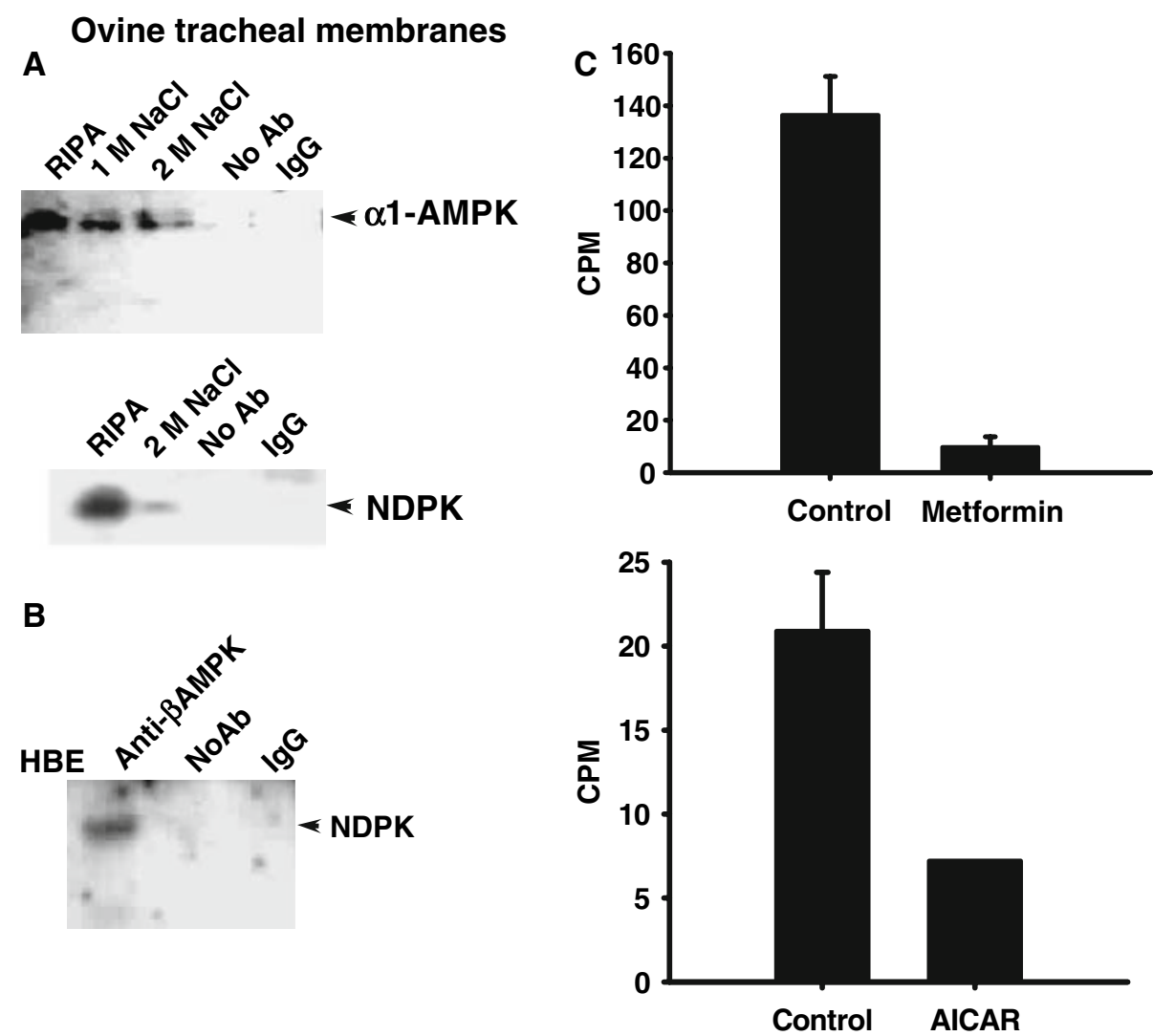

Fig. 5 Colocalisation of NDPK with AMPK. a Primary human airway biopsy cells stained with NDPK and $\alpha$-AMPK antibodies as indicated. Arrow denotes the position of the apical membrane. b As in 'A' using Calu- 3 cell line grown at airliquid interface on a permeable support. c As in 'A' but cells are stained with a T172 phosphospecific antibody to $\alpha$-AMPK
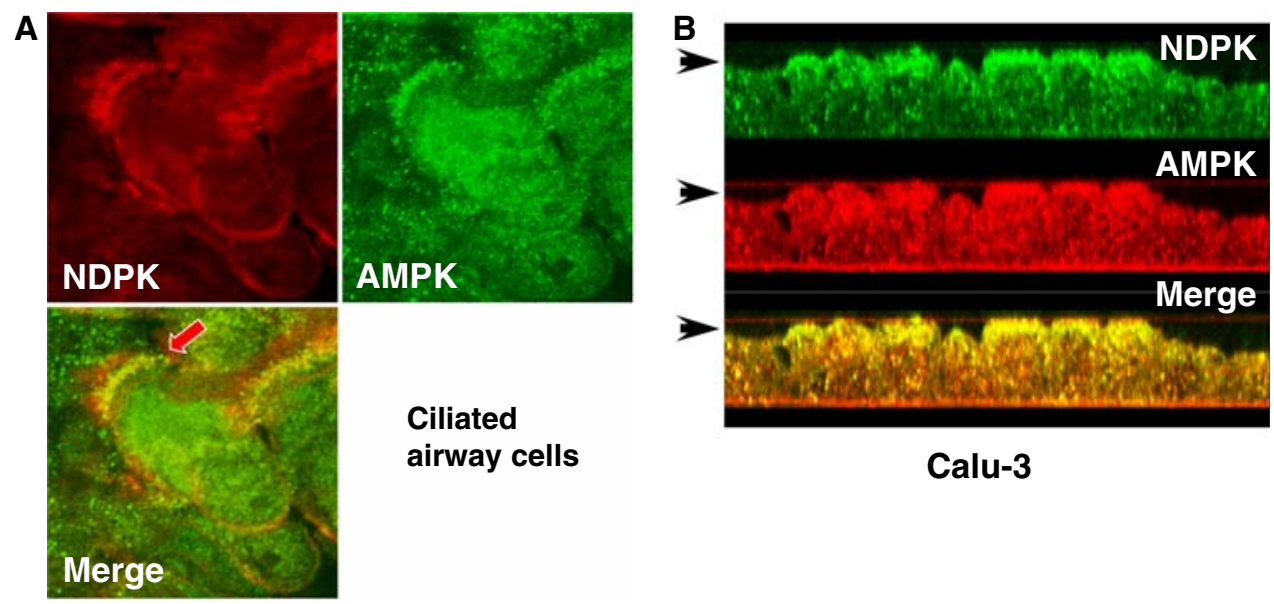

Calu-3

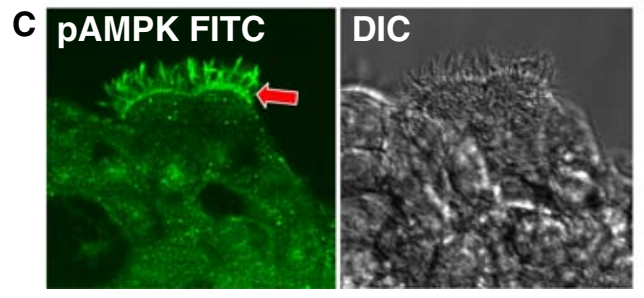

\section{Ciliated}

airway cells

panel) shows that when metformin (an activator of AMPK) was applied to the HBE cells, NDPK phosphorylation was almost abolished. Furthermore, a similar reduction in NDPK phosphorylation was observed in primary nasal brushings exposed to the cell-permeant AMP mimic, 5-aminoimidazole-4-carboxamide ribonucleoside (AICAR) (Fig. 4c bottom panel). Finally, the two proteins were found to colocalise at the apical membrane in primary 
A
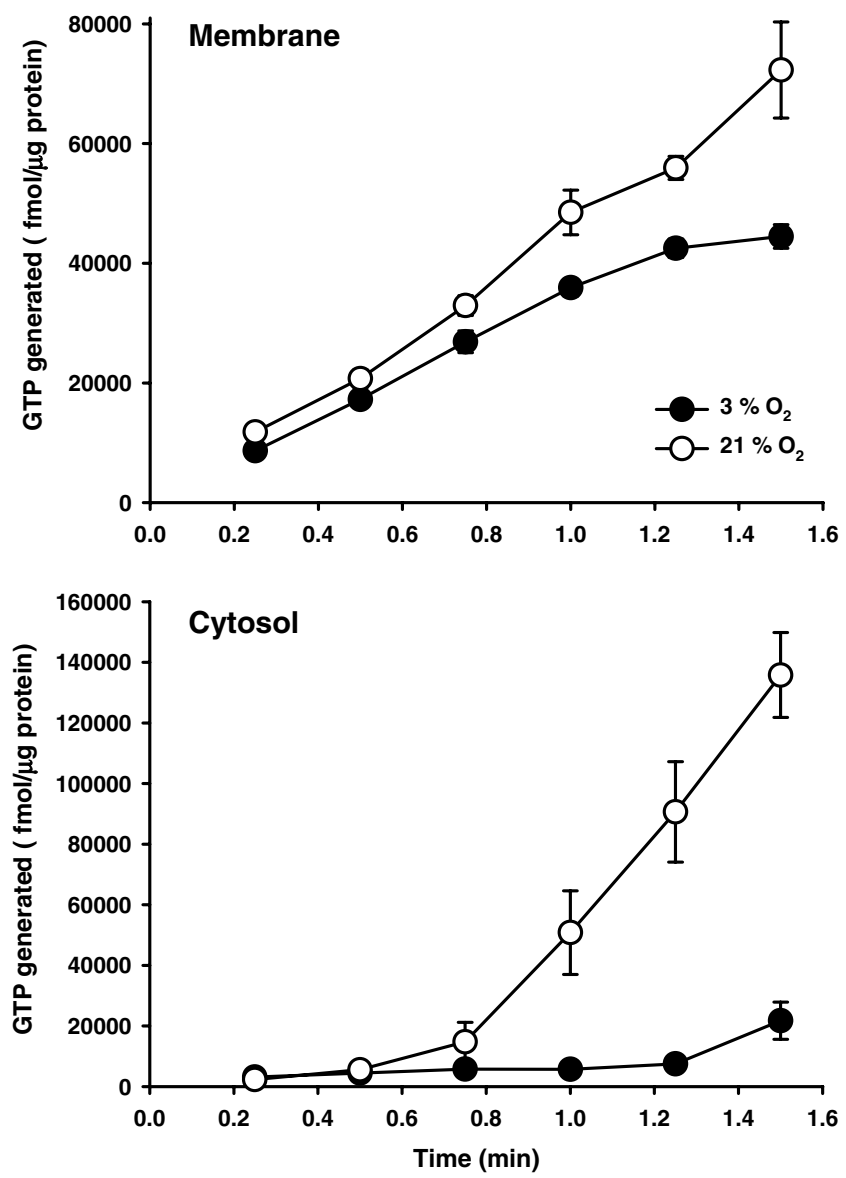

Fig. 6 Effect of hypoxia on NDPK function and colocalisation with AMPK. a NDPK activity in fractions from HBE cells grown in submersion culture under hypoxic (closed circles) or normoxic (open circles) conditions. Hypoxia decreased NDPK activity in both

human airway biopsies (Fig. 5a), a serous airway gland cell line (Fig. 5b) and HBE cells grown at air-liquid interface on permeable supports (Fig. 6c). Detection of the phosphorylated form of AMPK at T172 is considered to be a marker of activation. We observed that a T172 phosphospecific antibody localised to the apical membrane of human airway biopsies (Fig. 5c). In all instances, secondary antibody concentrations were such that there was no signal when primary antibody was absent.

Having found that activation of AMPK inhibits NDPK phosphorylation, we applied a more physiological stimulus using hypoxia, which is recognised to activate AMPK [20]. We observe that HBE cells exposed to low oxygen (3\% for $72 \mathrm{~h}$ ) manifested a decline in NDPK activity in both membrane and cytosol (Fig. 6a, cytosol greater than membrane). We observed a corresponding $50 \%$ reduction in membrane-bound total NDPK phosphorylation (1.5 min at $4^{\circ} \mathrm{C}$, Fig. $\left.6 \mathrm{~b}\right)$. In addition, the co-localisation of the two
B

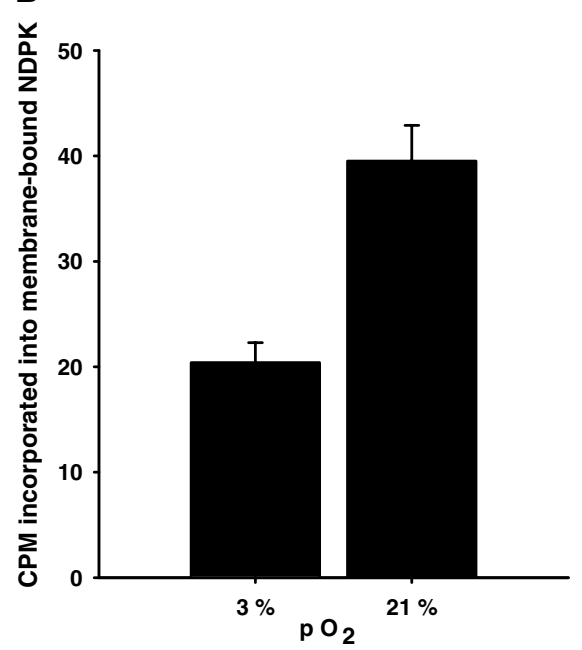

C $21 \%$

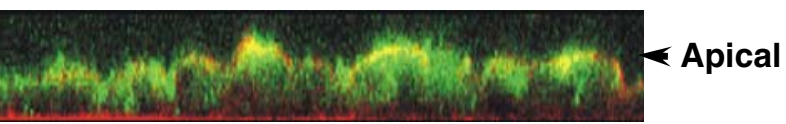

$3 \%$

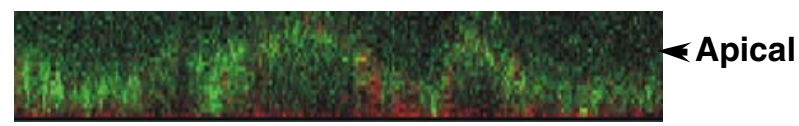

membrane and cytosol. b phosphorylation of NDPK declined by about 50\% under hypoxic conditions in HBE membranes, with a corresponding loss of colocalisation with AMPK in HBE cells grown at air-liquid interface (c)

proteins in the apical membrane was no longer observed under hypoxic conditions (Fig. 6c).

\section{Discussion}

We find that AMPK both co-precipitates and co-localises at the apical membrane with NDPK, and this is consistent across several different cell types, including primary human ciliated cells. We demonstrate that AMP differentially modulates the histidine and serine phosphorylation (pHis and pSer respectively) of NDPK that is bound to the epithelial membrane. Surprisingly, pSer (and its augmentation with [AMP]) is only apparent when GTP is the phosphate donor. In contrast, increasing [AMP] decreases pHis regardless of the phosphate donor (more apparent with ATP). The mechanism by which AMPK could induce this effect is not easy to reconcile because GTP is not 
utilised by AMPK. Thus the simple hypothesis that AMPK phosphorylates NDPK is not supported by our data. It remains possible that NDPK has serine autophosphorylation activity although it seems unlikely that such a mechanism would discriminate between nucleoside triphosphate donors, as we do not see this labelling with ATP, only with GTP. The alternative explanation is that a separate kinase, capable of utilising GTP, could be stimulated by AMP to phosphorylate NDPK. CK2, which is known to phosphorylate NDPK [3], can use GTP as well as ATP, however, the same problem arises in that the conditions under which CK2 might use GTP preferentially are not understood. Our data also challenge the simple idea that NDPK merely balances nucleotide pools indiscriminately. The combined data suggest that complex regulation of NDPK by several interacting kinases exists at the epithelial membrane. The presence of phospho-serine in NDPK has been ascribed to an artifactual transfer from high-energy phospho-histidine to an adjacent low energy serine, which, in some instances, is promoted by the presence of acid during the experimental process. However, our data show a differential generation of phosphoserine that cannot be attributed to experimental artefact.

We found an unexpected potent effect of GMP relative to AMP in its capacity to inhibit NDPK pHis content (Fig. 1) and that the AMPK activator, AICAR stimulated phosphorylation of a $37 \mathrm{kDa}$ protein (unpublished observation). We observe (Fig. 2) that when GTP is the phosphate donor, a $37 \mathrm{kDa}$ phosphoprotein is labelled. This is not observed with ATP. In a previous study [6], we found that the transfer of $\gamma$-labelled phosphate from GTP (probably via membrane-bound NDPK) to annexin I occurred differentially in the presence of two almost identical nonhydrolysable analogues of GTP (5'-guanylimidodiphosphate; GppNp and $\beta \gamma$ methyleneguanosine $5^{\prime}$-triphosphate; GppCp). The GppNp compound obliterated the transfer by competing with GTP as expected, but the GppCp effect was incomplete. Thus the complex of NDPK partners in this membrane can discriminate the structure of the terminal phosphate link in GTP. The effects of the equivalent adenosine compounds on transfer from GTP were different again, suggesting that ATP and GTP can be differentially used as phosphate donors, at least in this particular complex, and offering a much more subtle means of regulating nucleotide and membrane metabolism. The effect of [AMP] on NDPK is equally complex. For example, AMP is inhibitory to NDPK phosphorylation when intact membranes are studied (Fig. 1a). In contrast, when semi-purified, membrane-extracted NDPK is added to a precipitate of AMPK, AMP inhibition can be observed but AMP has no effect on the semi-purified NDPK preparation alone (data not shown). Thus, it is likely that the close association of both protein kinases is required for AMP-induced NDPK inhibition of phosphorylation. This idea is consistent with the observed inhibitory effects of metformin and AICAR on NDPK phosphorylation in intact cells (Fig. 4c).

Both co-immunoprecipitation and immunofluorescence techniques suggest that a complex exists between AMPK and NDPK in airway epithelial cells. Furthermore, under hypoxic conditions, their co-localisation in immunofluorescence disappears. Interestingly, several different methods of activating AMPK (AMP, AICAR, metformin and hypoxia) all result in decreased NDPK pHis phosphorylation. We note that resting cellular $\mathrm{pH}(\sim 6.9)$ lies close to the level where pHis becomes unstable and that hypoxia will make the cell even more acidic, creating an environment where protein modification by phosphohistidine could become more sensitive to regulation. We used a hypoxic insult to mimic this effect and found that NDPK function, measured by its ability to generate GTP, was oxygen sensitive (cytosolic NDPK is more responsive to hypoxia than the membrane-bound NDPK). AMPK would be active under these hypoxic conditions, however, as stated above; our data do not support the notion that AMPK phosphorylates NDPK directly.

Here we present evidence for complex regulation of NDPK activity by interacting kinases, oxygen tension and nucleotide species. GTP has an overwhelmingly important regulatory role at the membrane and our data suggest that NDPK function, within this complex of proteins, is differentially responsive to guanosine- and adenosine-containing nucleotides. We speculate that it may be possible for NDPK to switch function from phospho-transferase to histidine kinase because our data suggest that serine phosphorylation (from GTP) promotes kinase function whilst inhibiting transferase function. This is mirrored following activation of AMPK, but it remains to be seen whether these apparently independent regulatory mechanisms are interrelated.

Acknowledgements KJT was funded by the Wellcome Trust (GR061003FR and 069150/Z/02/Z).

\section{References}

1. Freije JM, Blay P, MacDonald NJ, Manrow RE, Steeg PS (1997) Site-directed mutation of Nm23-H1. Mutations lacking motility suppressive capacity upon transfection are deficient in histidinedependent protein phosphotransferase pathways in vitro. J Biol Chem 272:5525-5532. doi:10.1074/jbc.272.9.5525

2. MacDonald NJ, Freije JM, Stracke ML, Manrow RE, Steeg PS (1996) Site-directed mutagenesis of nm23-H1. Mutation of proline 96 or serine 120 abrogates its motility inhibitory activity upon transfection into human breast carcinoma cells. J Biol Chem 271:25107-25116. doi:10.1074/jbc.271.41.25107

3. Biondi RM, Engel M, Sauane M, Welter C, Issinger OG, Jimenez de Asua L, Passeron S (1996) Inhibition of nucleoside diphosphate kinase activity by in vitro phosphorylation by protein 
kinase CK2. Differential phosphorylation of NDP kinases in HeLa cells in culture. FEBS Lett 399:183-187. doi:10.1016/ S0014-5793(96)01299-9

4. Kowluru A (2003) Defective protein histidine phosphorylation in islets from the Goto-Kakizaki diabetic rat. Am J Physiol Endocrinol Metab 285:E498-E503

5. Winder WW, Hardie DG (1999) AMP-activated protein kinase, a metabolic master switch: possible roles in type 2 diabetes. Am J Physiol 277:E1-E10

6. Muimo R, Banner SJ, Marshall LJ, Mehta A (1998) Nucleoside diphosphate kinase and $\mathrm{Cl}^{-}$sensitive protein phosphorylation in apical membranes from ovine airway epithelium. Am J Respir Cell Mol Biol 18:270-278

7. Muimo R, Crawford RM, Mehta A (2006) Nucleoside diphosphate kinase A as a controller of AMP-kinase in airway epithelia. J Bioenerg Biomembr 38:181-187. doi:10.1007/s10863-0069033-2

8. Muimo R, Hornickova Z, Riemen CE, Gerke V, Matthews H, Mehta A (2000) Histidine phosphorylation of annexin I in airway epithelia. J Biol Chem 275:36632-36636. doi:10.1074/ jbc.M000829200

9. Marshall LJ, Muimo R, Riemen CE, Mehta A (1999) $\mathrm{Na}^{+}$and $\mathrm{K}^{+}$ regulate the phosphorylation state of nucleoside diphosphate kinase in human airway epithelium. Am J Physiol 276:C109C119

10. Strelkov SV, Perisic O, Webb PA, Williams RL (1995) The 1.9 A crystal structure of a nucleoside diphosphate kinase complex with adenosine $3^{\prime}, 5^{\prime}$-cyclic monophosphate: evidence for competitive inhibition. J Mol Biol 249:665-674. doi:10.1006/jmbi.1995.0327

11. Anciaux K, Van Dommelen K, Willems R, Roymans D, Slegers $\mathrm{H}$ (1997) Inhibition of nucleoside diphosphate kinase (NDPK/ nm23) by cAMP analogues. FEBS Lett 400:75-79. doi:10.1016/ S0014-5793(96)01358-0

12. Meggio F, Pinna LA (2003) One-thousand-and-one substrates of protein kinase CK2? FASEB J 17:349-368. doi:10.1096/fj. 02-0473rev
13. Carling D, Hardie DG (1989) The substrate and sequence specificity of the AMP-activated protein kinase. Phosphorylation of glycogen synthase and phosphorylase kinase. Biochim Biophys Acta 1012:81-86. doi:10.1016/0167-4889(89)90014-1

14. Hardie DG, Carling D (1997) The AMP-activated protein kinase-fuel gauge of the mammalian cell? Eur J Biochem 246: 259-273. doi:10.1111/j.1432-1033.1997.00259.x

15. Woods A, Salt I, Scott J, Hardie DG, Carling D (1996) The $\alpha 1$ and $\alpha 2$ isoforms of the AMP-activated protein kinase have similar activities in rat liver but exhibit differences in substrate specificity in vitro. FEBS Lett 397:347-351. doi:10.1016/S00145793(96)01209-4

16. Cozens AL, Yezzi MJ, Chin L, Simon EM, Friend DS, Gruenert DC (1991) Chloride ion transport in transformed normal and cystic fibrosis epithelial cells. Adv Exp Med Biol 290:187-194 discussion 194-6

17. Borthwick LA, Neal A, Hobson L, Gerke V, Robson L, Muimo R (2008) The annexin 2-S100A10 complex and its association with TRPV6 is regulated by cAMP/PKA/CnA in airway and gut epithelia. Cell Calcium 44:147-157. doi:10.1016/j.ceca.2007.11.001

18. Treharne KJ, Marshall LJ, Mehta A (1994) A novel chloridedependent GTP-utilizing protein kinase in plasma membranes from human respiratory epithelium. Am J Physiol 267:L592-L601

19. Hardie DG, Carling D, Carlson M (1998) The AMP-activated/ SNF1 protein kinase subfamily: metabolic sensors of the eukaryotic cell? Annu Rev Biochem 67:821-855. doi:10.1146/ annurev.biochem.67.1.821

20. Wyatt CN, Mustard KJ, Pearson SA, Dallas ML, Atkinson L, Kumar P, Peers C, Hardie DG, Evans AM (2007) AMP-activated protein kinase mediates carotid body excitation by hypoxia. J Biol Chem 282:8092-8098. doi:10.1074/jbc.M608742200 